\section{Trasplante pulmonar ex vivo. Primera experiencia en Chile y Latinoamérica}

\author{
FELIPE UNDURRAGA M. ${ }^{1}$, JAVIER VEGA S. ${ }^{1}$, \\ CHRISTIAN FAJARDO J. ${ }^{\mathrm{a}}$, RODRIGO DÍAZ G. ${ }^{1}$, DAVID LAZO P. ${ }^{1}$, \\ HUASCAR RODRÍGUEZ G. ${ }^{\mathrm{b}}$, ELI VILLALABEITIA R. ${ }^{1}$, \\ EDGARDO SEPÚLVEDA V. ${ }^{1}$, JORGE RUFS B. ${ }^{1}$, JORGE DREYSE D. ${ }^{1}$, \\ FELIPE ALARCÓN O.c, JOSEFA VALENZUELA S.d, \\ NATALIA SEGUEL G. ${ }^{\mathrm{e}}$, ROBERTO CASTILLO R. ${ }^{1}$, \\ LORENA ARRAU P. ${ }^{\mathrm{f}}$, LORENA CALABRÁN R. ${ }^{1, \mathrm{f}}$, \\ PATRICIO RODRÍGUEZ D. ${ }^{1}$, JONATHAN C. YEUNG ${ }^{2}$
}

\section{Lung transplant with ex vivo lung perfusion, Chilean and Latin American first experience}

Background: The number of patients waiting for a lung transplant worldwide greatly exceeds the number of available donors. Ex vivo lung perfusion is a useful tool that allows marginal donor lungs to be evaluated and reconditioned for a successful lung transplantation. Aim: To describe the first Chilean and Latin American experience in ex vivo lung perfusion for marginal donor lungs before transplantation. Material and Methods: Descriptive analysis of all ex vivo lung perfusion conducted for marginal donor lungs at a private clinic, from April 2019 to October 2020. High risk donor lungs and rejected lungs from other transplantation centers were included. The "Toronto Protocol" was used for ex vivo lung perfusion. Donor lung characteristics and recipient outcomes were studied. Results: During the study period, five ex vivo lung perfusions were performed. All lungs were reconditioned and transplanted. No complications were associated. There were no primary graft dysfunctions and only one chronic allograft dysfunction. There was no mortality during the first year. The median arterial oxygen partial pressure/fractional inspired oxygen ratio increased from $266 \mathrm{~mm} \mathrm{Hg}$ in the donor lung to 419 after 3 hours of ex vivo lung perfusion $(p=0.043)$. Conclusions: ex vivo lung perfusion is a safe and useful tool that allows marginal donor lungs to be reconditioned and successfully transplanted.

(Rev Med Chile 2021; 149: 171-177)

Key words: Latin America; Lung; Lung Transplantation; Perfusion.

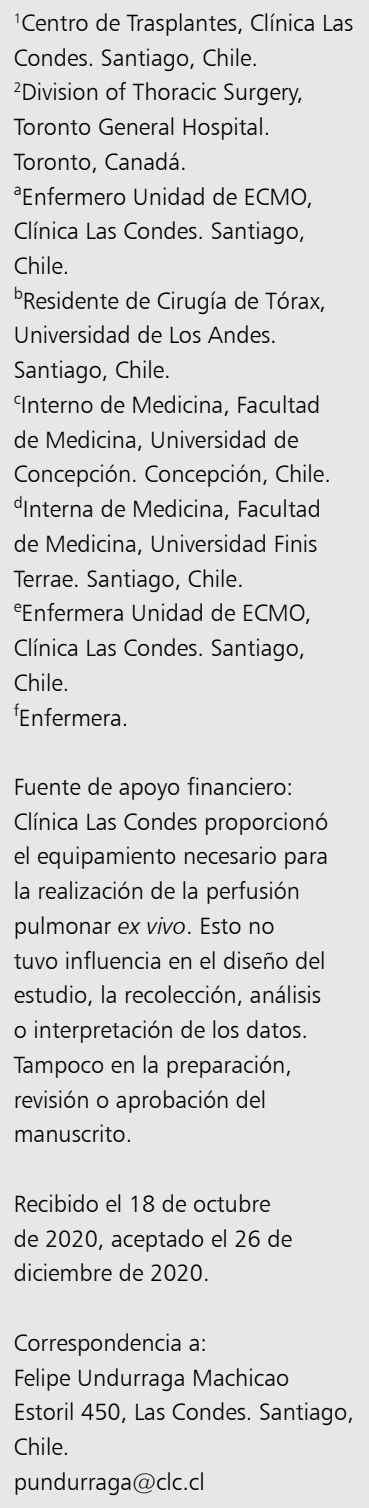

${ }^{1}$ Centro de Trasplantes, Clínica Las Condes. Santiago, Chile. 2Division of Thoracic Surgery, Toronto, Canadá.

aEnfermero Unidad de ECMO, Clinica Las Condes. Santiago,

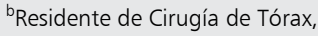
Universidad de Los Andes.

Santiago, Chile. Concepción. Concepción, Chile. IInterna de Medicina, Facultad de Medicina, Universidad Finis Terrae. Santiago, Chile.

'Enfermera Unidad de ECMO, Clínica Las Condes. Santiago, Chile.

Fuente de apoyo financiero: Clínica Las Condes proporcionó el equipamiento necesario para la realización de la perfusión pulmonar ex vivo. Esto no estudio, la recolección, análisis o interpretación de los datos. Tampoco en la preparación revisión o aprobación del Recibido el 18 de octubre de 2020, aceptado el 26 de diciembre de 2020
E 1 trasplante pulmonar es la única opción de tratamiento para pacientes seleccionados con enfermedades pulmonares en etapa terminal. En Chile la tasa de donación en los últimos 10 años ha oscilado entre 6 y 10 por millón de habitantes, siendo una de las más bajas de América ${ }^{2}$. Pese a que en el año 2019 hubo 175 donantes efectivos, solo se llevaron a cabo 21 trasplantes pulmonares. Esto no es infrecuente, considerando que $80 \%$ de los pulmones donados son rechazados por categorizarse como marginales o por problemas logísticos en su extracción ${ }^{1,3}$. A nivel mundial existe una diferencia importante entre el número de pacientes en lista de espera y los pulmones disponibles para ser donados, lo que determina una tasa de mortalidad en lista de espera de alrededor de $30 \%{ }^{4}$. La adopción de donantes con criterios extendidos, el trasplante monopulmonar o lobar, han sido las primeras herramientas utilizadas para disminuir esta brecha. Sin embargo 
en el año 2001 surgió una nueva alternativa, la técnica de perfusión pulmonar ex vivo (EVLP $)^{5}$. Esta consiste en perfundir y ventilar a normotermia los pulmones por varias horas dentro de un circuito aislado extracorpóreo, que permite la evaluación y optimización del órgano a trasplantar ${ }^{6}$.

El objetivo de este estudio es describir la primera experiencia nacional y latinoamericana en pulmones marginales sometidos a la técnica de EVLP que llegaron a ser utilizados en receptores.

\section{Material y Método}

Serie de casos, análisis descriptivo y retrospectivo de los pacientes trasplantados pulmonares, con técnica de perfusión pulmonar ex vivo, en Clínica Las Condes, desde abril de 2019 a octubre de 2020. Se incluyeron solo aquellos pulmones categorizados como marginales (mejor presión arterial de oxígeno/fracción inspirada de oxígeno $[\mathrm{PaFi}]<300 \mathrm{mmHg}$, edema pulmonar detectado en radiografía de tórax o durante la exploración quirúrgica, reclutamiento pulmonar inadecuado durante el procuramiento, historia dudosa de aspiración o transfusiones sanguíneas múltiples) y que fueron rechazados por todos los centros de trasplante nacionales. Se excluyeron aquellos pulmones de donantes con neumonias confirmadas, traumatismo grave (contusión en más de 1 lóbulo) o evidencia de aspiración de contenido gástrico.

\section{Técnica}

El procuramiento pulmonar se realizó según la técnica estándar ${ }^{7}$, la preservación del órgano con hipotermia de superficie $\left(4^{\circ} \mathrm{C}\right)$, el almacenaje en una solución de dextrano bajo en potasio (Perfadex $^{\oplus}$ ) y el transporte por vía aérea o terrestre. Una vez llegado el órgano a Clínica Las Condes, fue recibido y conectado a EVLP por un equipo multidisciplinario entrenado en dicha técnica. Para la EVLP se siguió estrictamente el "Protocolo Toronto". El cebado del circuito se llevó a cabo con 2 L de solución de Steen (Vitrolife ${ }^{\circledast}$ ) a la que se le adicionaron $500 \mathrm{mg}$ de metilprednisolona, $500 \mathrm{mg}$ de imipenem/cilastatina y 3.000 UI de heparina. El pulmón se transfirió a una cámara especial, se conectó el tronco de la arteria pulmonar a una cánula, al igual que el "cuff" de la aurícula izquierda. La tráquea se conectó a un tubo endotraqueal monolumen (Figura 1). Durante la conexión y llenado del árbol vascular pulmonar se desecharon $300 \mathrm{~mL}$ de solución y se inició la perfusión a $20^{\circ} \mathrm{C}$ con $10 \%$ del flujo sanguíneo calculado para el pulmón donado (40\% del gasto cardíaco calculado para el donante). Durante los primeros 50 min se aumentó el flujo y la temperatura paulatinamente hasta llegar a $37^{\circ} \mathrm{C}$ y a $100 \%$ del flujo calculado para el pulmón donado. En $32^{\circ} \mathrm{C}$, se inició la ventilación mecánica del pulmón y el flujo de gas al circuito de perfusión, con el objetivo de desoxigenar y aportar dióxido de carbono a la solución. La presión de arteria pulmonar media (PAPM) se mantuvo entre 7 y $15 \mathrm{mmHg}$ y la presión de la aurícula izquierda (PAI) entre 3 y 5 $\mathrm{mmHg}$. Se llevó a cabo una ventilación mecánica protectora con un volumen corriente (VC) de 7 $\mathrm{mL} / \mathrm{kg}$ (basada en el peso ideal del donante) a 7 respiraciones por minuto, con presión positiva al final de la espiración (PEEP) de $5 \mathrm{~cm} \mathrm{H}_{2} \mathrm{O}_{\text {y FiO}}$ de $21 \%$. El pH, $\mathrm{pCO}_{2}$, los electrolitos y la glucosa se mantuvieron en niveles fisiológicos. Los pulmones fueron reclutados cada 1 hora con una presión pico de vía aérea de $20 \mathrm{~cm} \mathrm{H}_{2} \mathrm{O}$. Para evaluar la función pulmonar, la $\mathrm{FiO}_{2}$ se aumentó a $100 \%$, el $\mathrm{VC}$ a $10 \mathrm{~mL} / \mathrm{kg}$ y la frecuencia respiratoria a 10 respiraciones por minuto, durante $5 \mathrm{~min}$. Cada una hora se realizó medición de PaFi en cada vena pulmonar, PAPM, compliance dinámica y presión pico de vía aérea. La radiografía al pulmón en EVLP (Figura 2) y fibrobroncoscopía se realizaron a la hora 1 y 3 . La decisión de trasplantar los pulmones sometidos a EVLP se tomó a la hora 3.

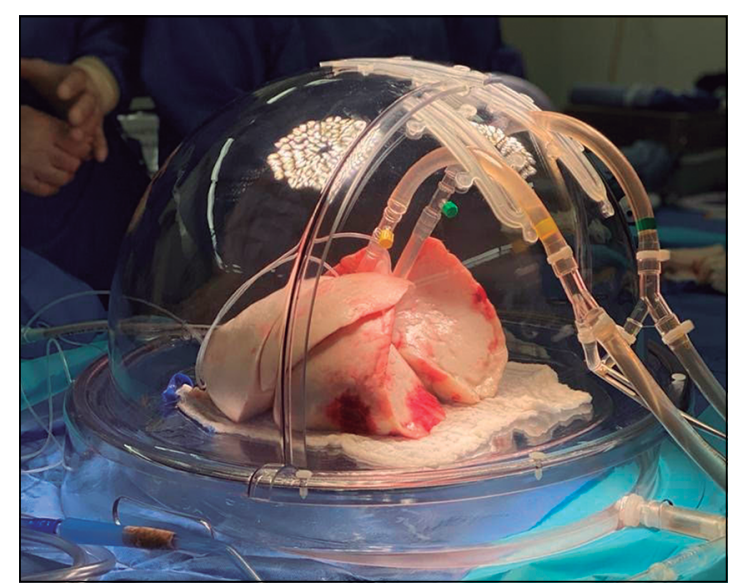

Figura 1. Pulmón dentro de una cámara adaptada para perfusión pulmonar ex vivo, conectado a una cánula arterial, auricular y a un tubo endotraqueal monolumen. 


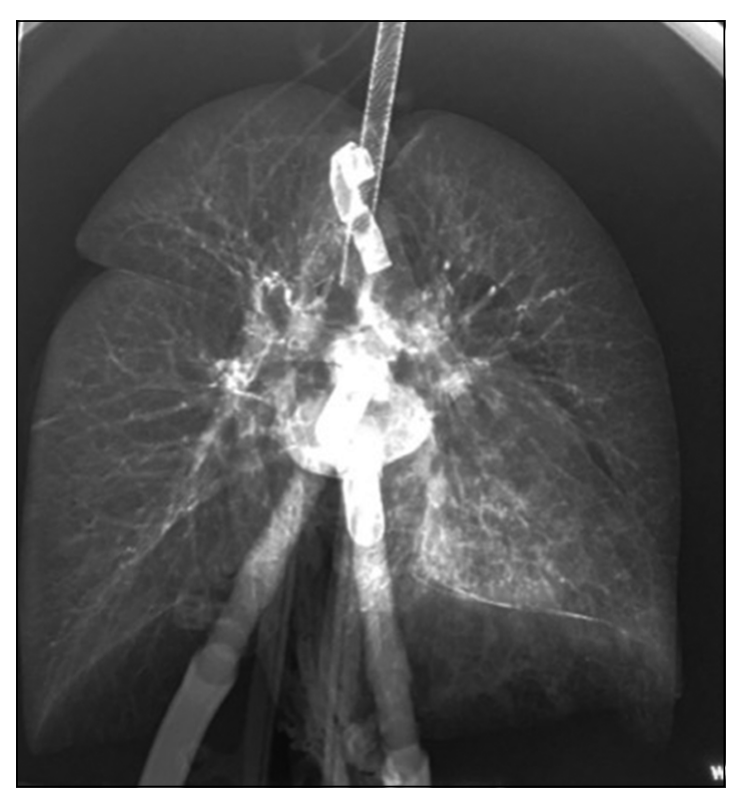

Figura 2. Radiografía pulmonar dentro de una cámara adaptada para perfusión pulmonar ex vivo.

Se debían cumplir los siguientes criterios: PaFi $>400 \mathrm{mmHg}$, mejoría o estabilidad de la PAPM, presión pico de la vía aérea, compliance dinámica y mejoría radiológica de los pulmones. Al final de la EVLP, los pulmones se enfriaron en el circuito en un período de $10 \mathrm{~min}$. Posteriormente se detuvo la perfusión y ventilación. Se realizó un pinzamiento traqueal para mantener los pulmones insuflados y estos se almacenaron en una solución de Perfadex ${ }^{\circledast}$ a $4^{\circ} \mathrm{C}$ hasta el momento de trasplante.

\section{Análisis estadístico}

Se consideraron variables del receptor y del órgano donado. Del receptor: edad, sexo, diagnóstico, tiempo en lista de espera, tipo de trasplante, días en oxigenación por membrana extracorpórea (ECMO) post trasplante, días en ventilación mecánica (VM) post trasplante, días en unidad de cuidados intensivos (UCI) post trasplante, días de hospitalización, complicaciones postoperatorias, disfunción primaria de injerto, rechazo crónico y supervivencia a corto plazo. Del órgano donado: tiempo de isquemia, PaFi, PAPM, PAI, compliance dinámica, presión pico de vía aérea y tiempo en EVLP. Se describieron las variables continuas como promedio y rango, y las variables categóricas como número y porcentaje. Se com- paró la PaFi promedio durante el procuramiento, 10 min previos al pinzamiento aórtico y la $\mathrm{PaFi}$ promedio registrada a hora 3 de EVLP, mediante la prueba de rangos de Wilcoxon para muestras relacionadas. Para el análisis estadístico se utilizó el programa SPSS ${ }^{\circledR} \mathrm{V} 25$ y se consideró significativo un valor $\mathrm{p}<0,05$.

El estudio contó con la aprobación del Comité de Ética de la Investigación de Clínica Las Condes.

\section{Resultados}

Durante el período estudiado, 5 pulmones fueron sometidos a EVLP. En 100\% de los casos se logró la optimización del órgano y trasplante, sin registrar complicaciones asociadas al procedimiento, ni relacionadas con el dispositivo. La edad promedio del receptor fue 38 años, siendo la mayoría de sexo masculino ${ }^{4}$. El diagnóstico predominante fue la fibrosis pulmonar y el tiempo promedio en lista de espera fueron 84 días (Tabla 1). Los días promedio en ECMO, VM post trasplante, UCI post trasplante y de hospitalización, fueron 3, 7, 16 y 53 respectivamente. Tres pacientes presentaron complicaciones postoperatorias, ninguno presentó disfunción primaria de injerto y uno presentó rechazo crónico. No hubo mortalidad a 30 días de seguimiento (Tabla 2).

\section{Tabla 1. Características demográficas de los pacientes sometidos a trasplante pulmonar ex vivo}

\begin{tabular}{|lc|}
\hline Característica & $\begin{array}{c}\text { Promedio } \\
\text { (rango) o } \\
\text { Número }\end{array}$ \\
\hline Edad (años) & $38(7-67)$ \\
\hline Sexo & \\
Masculino & 4 \\
Femenino & 1 \\
Diagnóstico & \\
Fibrosis pulmonar & 4 \\
Síndrome de bronquiolitis obliterante & 1 \\
Tiempo en lista de espera (días) & $84(2-381)$ \\
Tipo de trasplante & \\
Bipulmonar & 2 \\
Monopulmonar & 2 \\
Bilobar & 1 \\
\hline
\end{tabular}


Tabla 2. Resultados post quirúrgicos de los pacientes sometidos a trasplante pulmonar ex vivo

\begin{tabular}{|lc|}
\hline Característica & $\begin{array}{c}\text { Promedio } \\
\text { (rango) o } \\
\text { Número }\end{array}$ \\
\hline ECMO (días) & $3(2-6)$ \\
VM (días) & $7(0-21)$ \\
UCl (días) & $16(3-32)$ \\
Hospitalización (días) & $53(14-117)$ \\
Complicaciones post quirúrgicas & \\
Si & 3 \\
Trombosis venosa profunda & 1 \\
Sepsis por Citomegalovirus & 1 \\
Estenosis anastomosis arterial & 1 \\
Rechazo crónico & \\
Si & 1 \\
Supervivencia (\%) & \\
30 días & 100 \\
\hline
\end{tabular}

*ECMO: oxigenación por membrana extracorpórea, VM: ventilación mecánica, UCl: unidad de cuidados intensivos.

Con respecto al órgano donado, el tiempo promedio de isquemia previo a EVLP fueron $294 \mathrm{~min}$. La PaFi, PAPM, PAI, compliance dinámica y presión pico de vía aérea promedio a la tercera hora de EVLP fue de $419 \mathrm{mmHg}, 8 \mathrm{mmHg}, 5 \mathrm{mmHg}$, $101 \mathrm{~mL} / \mathrm{cm} \mathrm{H}_{2} \mathrm{O}$ y $13 \mathrm{~cm} \mathrm{H} \mathrm{H}_{2} \mathrm{O}$, respectivamente (Figura 3). Al comparar el índice PaFi promedio obtenido durante el procuramiento, $10 \mathrm{~min}$ previos al pinzamiento aórtico $(266 \mathrm{mmHg})$ con el obtenido a la hora 3 de EVLP (419 mmHg), existió una diferencia estadísticamente significativa $(\mathrm{p}=0,043)$ (Figura 4). El tiempo promedio en EVLP fueron 250 min (Tabla 3 ).

\section{Discusión}

En Chile y en el mundo, el trasplante pulmonar esta limitado por la escasez de donantes y las complicaciones que se desencadenan posterior al implante, tales como la disfunción primaria de injerto ${ }^{8}$.

A lo largo de los años, la hipotermia ha sido la principal técnica para preservar los órganos
Tabla 3. Parámetros fisiológicos evaluados durante la perfusión pulmonar ex vivo

\begin{tabular}{|c|c|}
\hline Parámetro & $\begin{array}{c}\text { Promedio } \\
\text { (rango) o } \\
\text { Número }\end{array}$ \\
\hline Tiempo de isquemia previo a EVLP (min) & $294(205-450)$ \\
\hline \multicolumn{2}{|l|}{ PaFi $(\mathrm{mmHg})$} \\
\hline Previo a EVLP & $266(150-350)$ \\
\hline Hora 1 & $476(410-577)$ \\
\hline Hora 2 & $419(337-500)$ \\
\hline Hora 3 & $419(357-524)$ \\
\hline \multicolumn{2}{|l|}{ PAPM (mmHg) } \\
\hline Hora 1 & $8(6-10)$ \\
\hline Hora 2 & $8(5-12)$ \\
\hline Hora 3 & $8(6-9)$ \\
\hline \multicolumn{2}{|l|}{ PAl $(\mathrm{mmHg})$} \\
\hline Hora 1 & $5(2-7)$ \\
\hline Hora 2 & $5(1-7)$ \\
\hline Hora 3 & $5(2-7)$ \\
\hline \multicolumn{2}{|l|}{ Compliance dinámica $\left(\mathrm{mL} / \mathrm{cm} \mathrm{H}_{2} \mathrm{O}\right)$} \\
\hline Hora 1 & $96(70-125)$ \\
\hline Hora 2 & $120(81-124)$ \\
\hline Hora 3 & $101(85-120)$ \\
\hline \multicolumn{2}{|l|}{ Presión pico de vía aérea $\left(\mathrm{cm} \mathrm{H}_{2} \mathrm{O}\right)$} \\
\hline Hora 1 & $12(10-17)$ \\
\hline Hora 2 & $11(8-14)$ \\
\hline Hora 3 & $13(11-15)$ \\
\hline Tiempo en EVLP (min) & $250(211-310)$ \\
\hline
\end{tabular}

*EVLP: perfusión pulmonar ex vivo; PaFi: presión arterial de oxígeno/fracción inspirada de oxígeno; PAPM: presión de arteria pulmonar media; PAI: presión de aurícula izquierda.

donados, ya que reduce el metabolismo celular, los requerimientos de oxígeno y el consumo de nutrientes esenciales. Sin embargo, este método no permite evaluar la fisiología, identificar las noxas, ni monitorizar la recuperación del órgano a trasplantar. El no pesquisar una injuria puede manifestarse en el período post trasplante como una disfunción primaria de injerto9.

La técnica de EVLP permite preservar el metabolismo, la homeostasis pulmonar y actúa como plataforma para la evaluación y tratamiento del órgano donado ${ }^{8}$.

Ya se ha demostrado que los pulmones donados, categorizados como marginales, que son sometidos a EVLP previo al trasplante, tienen 
a)

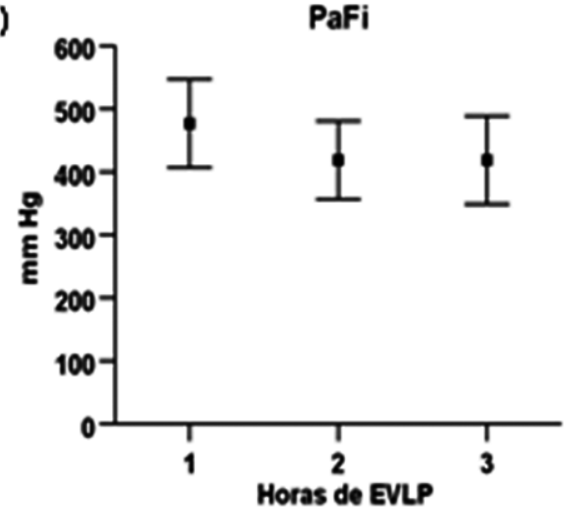

c)

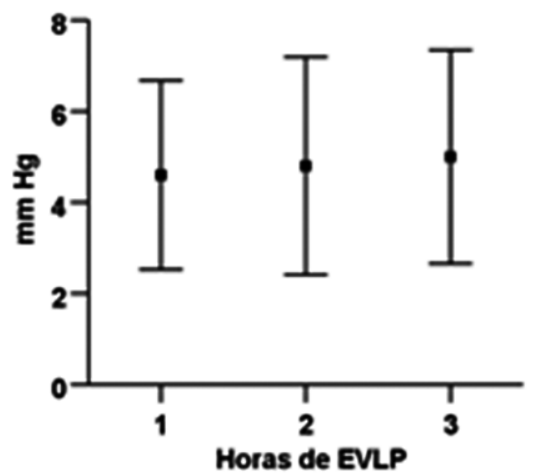

b) Presión de arteria pulmonar media

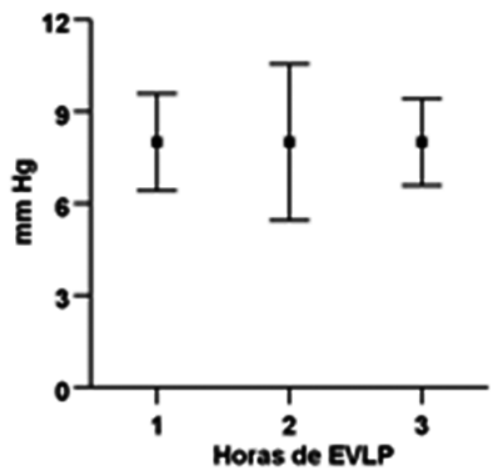

d) Compliance dinámica

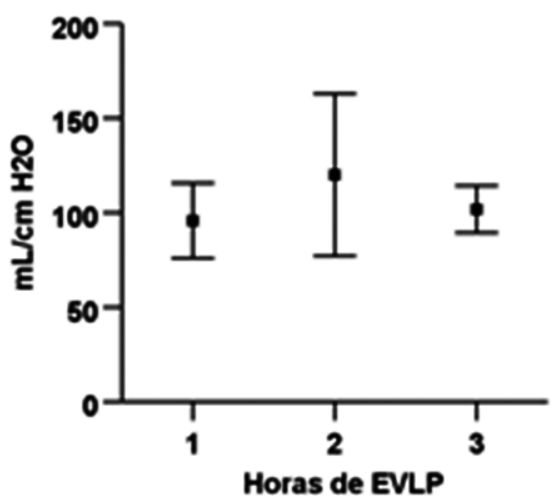

e) Presión pico de la vía aérea

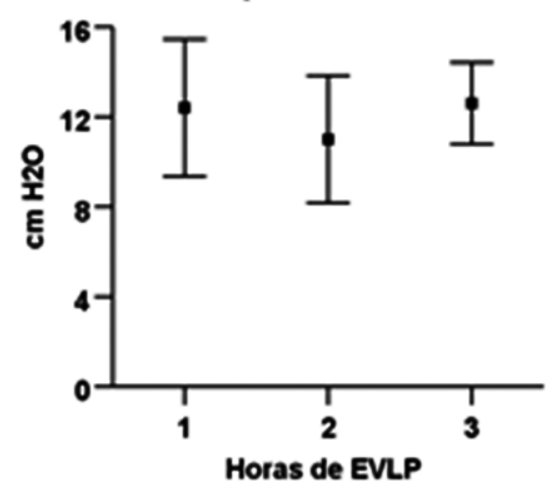

Figura 3. Función pulmonar evaluada a la hora 1, 2 y 3 durante la prefusión pulmonar ex vivo. (a) Índice presión arterial de oxígeno/fracción inspirada de oxígeno; (b) Presión de arteria pulmonar media; (c) Presión de aurícula izquierda; (d) Compliance dinámica; (e) Presión pico de la vía aérea. *PaFi: índice presión arterial de oxígeno/fracción inspirada de oxígeno; EVLP: perfusión pulmonar ex vivo. 


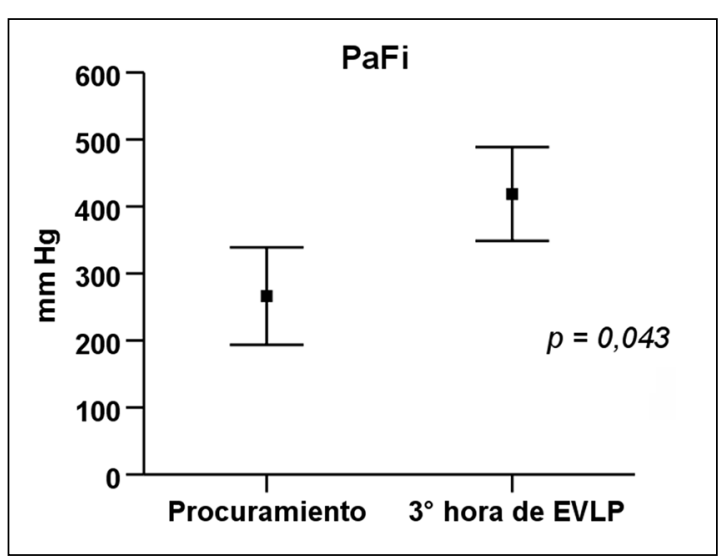

Figura 4. Diferencia entre índice presión arterial de oxígeno/ fracción inspirada de oxígeno (PaFi) durante procuramiento, 10 minutos previos al pinzamiento aórtico y la hora 3 de perfusión pulmonar ex vivo (EVLP). *PaFi: índice presión arterial de oxígeno/fracción inspirada de oxígeno; EVLP: perfusión pulmonar ex vivo.

resultados comparables con aquellos no marginales $^{1,10}$. Estas fueron las principales razones para que nuestro equipo decidiera adoptar esta técnica como una herramienta más en el arsenal terapéutico para el manejo de los pacientes con enfermedades pulmonares en fase terminal.

El poder controlar el órgano de forma más detallada permite rescatar un pulmón e incluso un lóbulo, en vez de desechar ambos pulmones en caso de un deterioro localizado. En nuestra serie se realizaron 2 trasplantes monopulmonares y uno bilobar.

En relación a las patologías que motivaron al trasplante, cabe destacar que uno de los casos de fibrosis pulmonar fue debida a infección por SARS$\mathrm{CoV}-2$. Dicho paciente fue uno de los primeros a nivel mundial en ser trasplantado de pulmón.

En un metanálisis y revisión sistemática publicada recientemente, el uso de ECMO en el postoperatorio de los pacientes trasplantados con técnica de EVLP, fue 3,72 veces mayor ( $95 \%$ IC, $0,83-16,66 ; p=0,09)$ que aquellos sin EVLP. También el uso de VM fue 2,17 días más prolongado (95\% IC, 0,63-4,96; $p=0,13)$. Sin embargo ninguno de estos dos parámetros fue estadísticamente significativo $^{11}$. En nuestra serie los días de ECMO y VM promedio fueron solo 3 y 7 , respectivamente.

Resultados similares se vieron en relación a la estadía en UCI y hospitalaria. Si bien en el primer grupo la estadía en UCI fue 2,56 días mayor (95\% IC, 2,29-7,42; $\mathrm{p}=0,30)$ y la estadía hospitalaria 3,15 días mayor (95\% IC, 0,99-7,29; $\mathrm{p}=0,14$ ), ambas diferencias no fueron estadísticamente significativas ${ }^{11}$.

Tsuang et al, demostraron mediante un metanálisis y revisión sistemática, que los pacientes trasplantados con técnica de EVLP no presentaban diferencias en relación a la disfunción primaria de injerto grado 3 a las 72 h (RR: 0,99, 95\% IC $0,56-1,75)^{12}$. Incluso el grupo de Toronto, uno de los que posee mayor experiencia en esta técnica, en un ensayo clínico prospectivo, reportó $15 \%$ de disfunción primaria de injerto grado 3 a las $72 \mathrm{~h}$, en los pulmones sometidos a EVLP, comparado con $30 \%$ presente en el grupo control, sin embargo esta diferencia no fue estadísticamente significativa $(\mathrm{p}=0,11)^{8}$. En nuestra serie ningún paciente presentó disfunción primaria de injerto.

Otro aspecto importante a considerar es el desarrollo de rechazo crónico. A la fecha no se ha demostrado que los pacientes a cuyos pulmones se les sometió a la técnica de EVLP presenten mayor incidencia de rechazo crónico.

Una cohorte retrospectiva de 706 pacientes, con seguimiento a 10 años, presentada por Divithotawela et al, confirma esto ${ }^{10}$. El grupo sueco de Ghaidan et al, también comparó la presencia de rechazo crónico a 10 años de seguimiento entre aquellos sometidos a EVLP y trasplante convencional. Si bien el número de pacientes era menor (21), los resultados fueron similares ${ }^{13}$. En nuestra serie, pese a que el seguimiento es mucho menor, dada la reciente implementación de esta técnica, hasta el momento solo hay un paciente que presentó rechazo crónico.

Con respecto a la supervivencia, el grupo canadiense de Cypel et al, demostraron que no existen diferencias estadísticamente significativas en la supervivencia a corto y largo plazo, entre aquellos sometidos a EVLP y trasplante convencional (HR: 0,07 , IC 95\% 0,75-1,27; $p=0,83$ ), siendo esta una de las series más grandes a nivel mundial ${ }^{14}$. Es nuestro estudio, el seguimiento es a corto plazo. Sin embargo, a la fecha, no registramos fallecidos.

Uno de los hallazgos más importantes durante la EVLP fue que al comparar el índice PaFi promedio obtenido durante el procuramiento, $10 \mathrm{~min}$ previos al pinzamiento aórtico $(266 \mathrm{mmHg})$ con el obtenido a la hora 3 de EVLP $(419 \mathrm{mmHg})$, existió una diferencia estadísticamente signifi- 
cativa $(\mathrm{p}=0,043)$. Esto refleja un aumento del reclutamiento pulmonar y una optimización de la relación ventilación/perfusión, lo que repercute positivamente en el órgano a trasplantar.

Una de las limitaciones de este estudio es el número reducido de pacientes, lo que se explica por la baja tasa de donación efectiva y la reciente aplicación de la técnica. Esto repercute inevitablemente en el seguimiento, que al ser breve, limita el estudio de las complicaciones a largo plazo (ej: rechazo crónico). De momento solo contamos con 18 meses de seguimiento para el primer caso.

A modo de conclusión la técnica de EVLP se presenta como una herramienta válida y segura para pulmones marginales, permitiendo su optimización para ser implantados. Es posible implementar un programa de ex vivo en Chile, considerando la necesidad de ensamblar un equipo multidisciplinario con entrenamiento adecuado, acceso a insumos e infraestructura específica y con criterios estrictos de selección del órgano donante.

\section{Referencias}

1. Cypel M, Yeung J, Machuca T, Chen M, Singer L, Yasufuku K, et al. Experience with the first 50 ex vivo lung perfusions in clinical transplantation. J Thorac Cardiovasc Surg. 2012; 144: 1200-7.

2. Hepp J, Beca JP, Morán S, Roessler E, Uribe M, Palacios JM. Donación y trasplante de órganos: propuesta desde la Academia Chilena de Medicina. Rev Med Chile 2020; 148: 381-6.

3. Estadísticas actuales de trasplantes, Ministerio de Salud, Chile. Disponible en: https://yodonovida.minsal.cl/ statistics/public/show/25 [Consultado el 5 de octubre de 2020].

4. Gottlieb J. Lung allocation. J Thorac Dis. 2017; 9: 26704.

5. Steen S, Sjoberg T, Pierre L, Liao Q, Eriksson L, Algots- son L. Transplantation of lungs from a non-heart-beating donor. Lancet. 2001; 357: 825-9.

6. Possoz J, Neyrinck A, Van Raemdonck. Ex vivo lung perfusion prior to transplantation: an overview of $\mathrm{cu}$ rrent clinical practice worldwide. J Thorac Dis. 2019; 11: 1635-50.

7. Sundaresan S, Trachiotis G, Aoe M, Patterson GA, Cooper J. Donor Lung Procurement: Assessment and Operative Technique. Ann Thorac Surg. 1993; 56: 140913.

8. Cypel M, Yeung J, Liu M, Anraku M, Chen F, Karolak W, et al. Normothermic Ex Vivo Lung Perfusion in Clinical Lung Transplantation. N Engl J Med. 2011; 364: 1431-40.

9. Reeb J, Cypel M. Ex vivo lung perfusion. Clin Transplant. 2016; 30: 183-94.

10. Divithotawela C, Cypel M, Martinu T, Singer L, Binnie $\mathrm{M}$, Chow CW, et al. Long-term outcomes of lung transplant with ex vivo lung perfusion. JAMA Surg. 2019; 154 (12): 1143-50.

11. Tian D, Wang Y, Shiiya H, Sun CB, Uemura Y, Sato M, et al. Outcomes of marginal donors for lung transplantation after ex vivo lung perfusion: A systematic review and meta-analysis. J Thorac Cardiovasc Surg. 2020: 159: 720-30.

12. Tsuang WM, Okamoto T, Morgan RL, Flack-Ytter Y, Budev MM, McCurry KR. Ex vivo lung perfusion resuscitated donor lungs and subsequent acute rejection and airway complications: a systematic review and meta-analysis. J Heart Lung Transplant. 2020; 39: S371.

13. Ghaidan H, Fakhro M, Andreasson J, Pierre L, Ingemansson R, Lindstedt S. Ten year follow-up of lung transplantations using initially rejected donor lungs after reconditioning using ex vivo lung perfusion. J Cardiothorac Surg. 2019; 14: 125.

14. Cypel M, Yeung J, Danahoe L, Chen M, Zamel R, Hoetzenecker $\mathrm{K}$, et al. Normothermic ex vivo lung perfusion: does the indication impact organ utilization and patient outcomes after transplantation? J Thorac Cardiovasc Surg. 2020; 159: 346-55.e1. 